\title{
Could embryonic diapause facilitate conservation of endangered species?
}

\author{
J Wauters ${ }^{1,2,3}$, K Jewgenow ${ }^{1}$, F Göritz ${ }^{4}$ and T B Hildebrandt ${ }^{4}$ \\ ${ }^{1}$ Department of Reproductive Biology, Leibnitz Institute for Zoo and Wildlife Research, \\ Germany \\ ${ }^{2}$ Laboratory of Chemical Analysis, Department of Veterinary Public Health and Food Safety, \\ Faculty of Veterinary Medicine, Ghent University, Belgium \\ ${ }^{3}$ Pairi Daiza Foundation - Pairi Daiza, Belgium \\ ${ }^{4}$ Department of Reproductive Management, Leibnitz Institute for Zoo and Wildlife \\ Research, Germany
}

Corresponding author e-mail: wauters@izw-berlin.de

\begin{abstract}
During embryonic diapause, the development of the embryo is paused shortly after conception by metabolic or seasonal factors. When conditions become favorable, the embryo will resume development after reactivation by the maternal reproductive system. Inducing this resting state in in vivo or in vitro manipulated embryos may offer invaluable advantages in the long-term storage of embryos, therefore offering a potential worthwhile and novel alternative in assisted reproduction compared to the currently broadly explored cryopreservation.

In this reflection paper, a discussion is launched on how diapause-induced embryo preservation may support breeding programs of threatened and endangered wildlife species. Detailed research on the pathways behind initiation and maintenance of the dormant state of a blastocyst should be the first priority to facilitate the introduction of embryonic diapause as a novel tool in assisted reproduction. In addition, the examples of giant pandas are used to discuss how research on diapause mechanism can be launched within captive breeding programs to better facilitate genetic biodiversity management in species that express diapause.
\end{abstract}

\section{Background}

Embryonic diapause, with arrested/delayed development of the embryo prior to implantation/attachment, occurs in more than 130 out of 2355 observed Eutherian and Marsupial species, belonging to at least 20 different families [1]. The phenomenon was first observed in roe deer in 1843, however, nearly 2 centuries later, the condition in roe deer is speculated to be delayed development rather than delayed implantation in this species $[2,3]$.

Focusing on true diapause, two major mechanisms are described: facultative diapause, mainly driven by metabolic parameters, and thus not occurring in every gestation, and obligate diapause, known to be dictated by seasonal factors, and therefore obligatory. The environmental, maternal and potentially embryonic triggers and resulting pathways behind the onset, maintenance and escape from diapause are not yet fully understood, but are clearly demonstrated to be different between species and between facultative and obligate diapause models. This is also reflected in a significant variation in histological and morphological appearances of the resting blastocyst, with carnivore paused blastocysts showing the highest cell count in the inner cell mass [4]. 
The best described models, but still under investigation, are the facultative model in mice (Mus musculus), the obligatory diapause in the mink (Neovison vison) and the intriguing combination model in the tammar wallaby (Macropus eugenii).

Metabolic stress induced by lactation is the driving factor in mouse diapause when the mouse conceives during postparturial estrus. This prevents the pre-implantation surge of estradiol, resulting in arrested development of the blastocyst, which first emerges from the zona pellucida. After hatching it aligns with the endometrial epithelium in the endometrial crypts without implanting until reactivation. A recent study of Kamemizu et al. [5] reported a cell-stage of $141.9+$ 20.3 in the diapaused embryo at D4.5 post coitum, which is in accordance with earlier studies. Reactivation occurs rapidly after a surge in estrogen in a progesterone-primed uterus $[4,5,6]$.

In most carnivores, including species of the families Ursidae, Mephitidae, Mustelidae, Otariidae and Phocidae, seasonal factors are regulating the embryonic diapause. Carnivore blastocysts contain a higher and more variable number of cells $(>200)$ and the embryo typically remains encapsulated in the zona pellucida, additionally shielded by 2 layers of glycoprotein $[4,7]$. A photoperiod trigger is the proximal stimulus for reactivation eventually leading to a prolactin (and LH)-induced increase in progesterone that allows resumption of embryonic development and supports subsequent attachment approximately 13 days later.

In the marsupial model, embryonic development is first delayed by suckling of the freshly born pouch young, since mating and conception are occurring immediately post-partum with only one exception, the swamp wallaby (Wallabia bicolor) which has a pre-partum estrus [8].The young leaves the pouch after approximately eight months. However, the blastocyst will not resume development until approximately three months later, when daylength is shortening. The paused blastocyst contains 60-100 cells, does not show an inner cell mass and is surrounded by the zona pellucida supplemented with two glycoprotein layers. In this model, the corpus luteum is inhibited by prolactin, first metabolically (suckling) and then seasonally induced. Reactivation is slow, as it only occurs after a three-day removal of the prolactin-induced inhibition, e.g. by decreasing daylength or early loss of the pouch young [1,9-11].

In all models, the exact pathways involved in embryonic arrest and its maintenance are less extensively studied compared to reactivation, despite having the potential for use in novel cancer therapies (growth inhibition) and in assisted reproduction. This paper will be focusing on how breakthroughs in research on embryonic diapause may be facilitating the conservation of threatened and endangered species with the general potential to revolutionize aspects of biobanking. A breakthrough would not only allow a more gentle preservation technique for sensitive tissue but would also substantially reduce the carbon foot print of biobanks in general.

\section{Discussion}

\section{Diapause-induced embryo preservation offers an alternative for cryopreservation}

Given the rapidly declining numbers of individuals for many wildlife species, assisted reproduction technologies (ARTs) have become increasingly relevant in wildlife conservation. ART allows not only to artificially advance the number of offspring, but also provides a tool to protect the genetic quality of the remaining population, particularly of the captive held fraction of specific populations across borders [12-17].

One of the most achievable approaches in the reproductive management of wildlife is cryopreservation of predominantly primordial reproductive cells, gametes and embryos [18-21]. Cryopreservation implies creating the possibility for long-term storage by introducing sub-zero temperatures to the cells, slowing down their metabolism - and thus energy needs - significantly. This technique is promising and shown to be successful, but has its downsides. Particularly slow- 
freezing causes physical (ice crystals) and chemical (osmotic) damage to the cells, and cannot be achieved successfully without strictly controlling the temperature reductions and adding cryoprotectants. Vitrification, or enhanced cryopreservation, by rapid cooling and liquid solidification, provided another breakthrough, however it remains a challenge in particular - even domestic - species, in which the technique fails, particularly in oocytes and embryos with high intracellular lipid content [22,23] or with high sensitivity towards cryoprotectants [24].

The damaging effects or limited applicability of cryopreservation for long-term storage of embryos could be bypassed if the freezing step could be omitted. The question therefore rises whether bringing the embryo in a dormant state to be transferred and reactivated at any later point in time, would be possible, even for non-diapausing animals.

Since the exact mechanisms behind the arrest in embryonic development are generally unknown, in vitro induction of diapause is currently not applicable. Nevertheless, mink blastocysts yielded from the uterus in a stage of delayed development were shown to withstand further in vitro culturing, while coculturing with uterine cells additionally increased the length and rate of survival [25]. In vitro survival of delayed embryos thus certainly seems to offer opportunities for diapausing species.

Interestingly, Ptak et al. [26] demonstrated the inherent potential of blastocysts of non-diapausing animals to enter growth arrest if transferred into a diapause-induced uterus. The latter is fairly easy established in mice uteri by ovariectomy shortly after ovulation. In this study, transferred sheep blastocysts remained in diapause during one week, were then flushed out from the mouse uteri for further in vitro development, or were transferred to a recipient sheep. In both cases, the blastocyst regained successful development, leading to birth after transferring a dormant blastocyst.

Arrest in non-diapausing blastocysts cannot be profitably induced or maintained artificially in vitro, despite potential indications for decelerated embryonic development, possibly caused by suboptimal culture conditions $[27,28]$. Thus, such an induced-dormancy/mouse-carrier system might offer new advantages to increase the life span of - for example - in vitro fertilized oocytes that - for whatever reason - cannot be transferred within the right timeframe. This would occur, for example, after the sudden death of a valuable individual with no cycling conspecific short-term available for embryo transfer.

\section{Diapause and pseudopregnancy exploited as a tool for genetic diversity management}

The most prominent endangered species which express diapause is the giant panda. There is an opportunity to elucidate the specific mechanism of diapause in giant pandas in order to facilitate assisted reproduction techniques and improve genetic diversity management in captivity. The suggested approach might serve as a blueprint for other species.

In giant pandas, the recognition of diapause is complicated by the obligate occurrence of pseudopregnancy in ovulated, but non-pregnant females [29]. After ovulation, the corpus luteum will undergo a dormancy phase of several months before reactivation, regardless of whether conception has occurred. The latter is accompanied by a primary and secondary increase in progesterone, respectively [30]. In this species, living on the edge of its metabolic capacities due to its evolutionary switch from carnivore to herbivore [31], two important assumptions need to be highlighted. Firstly, as described for other bear species, in all ovulated giant pandas the reproductive system is being pre-programmed for potential pregnancy [32,33]. Hence, no differences in hormone profiles have so far been observed between pregnant and pseudopregnant animals until after the window of implantation/attachment (see Figure 1) [34], in contrast to the later stage of the active luteal phase [35]. 


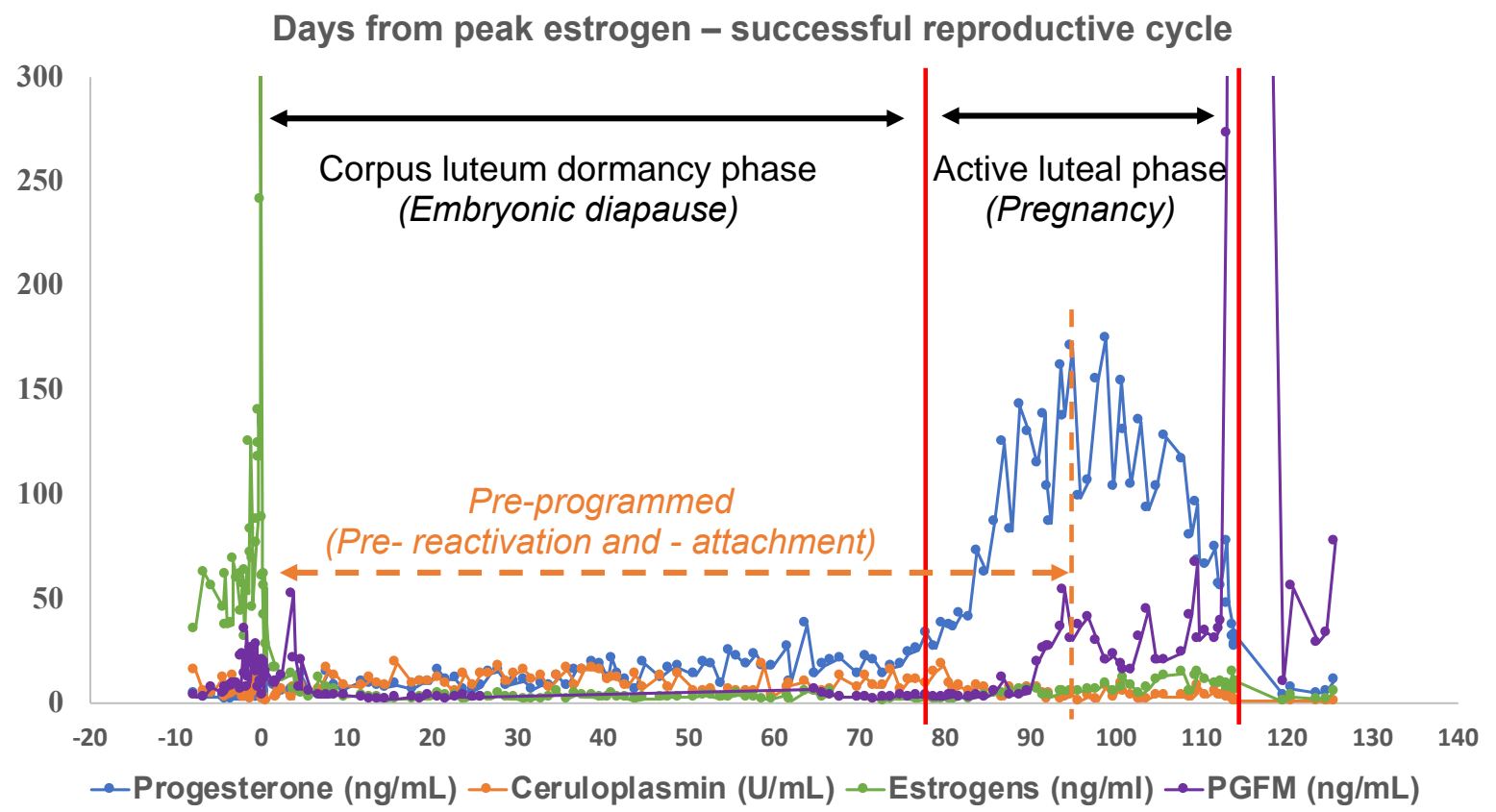

Figure 1 Overview of a successful cycle in a female giant panda. Monitoring was performed based on urinary samples corrected for urinary specific gravity. Four markers were observed: progesterone, estrogens, ceruloplasmin, PGFM (=13,14-dihydro-15-keto-PGF2alpha), as a measure of the endocrine status.

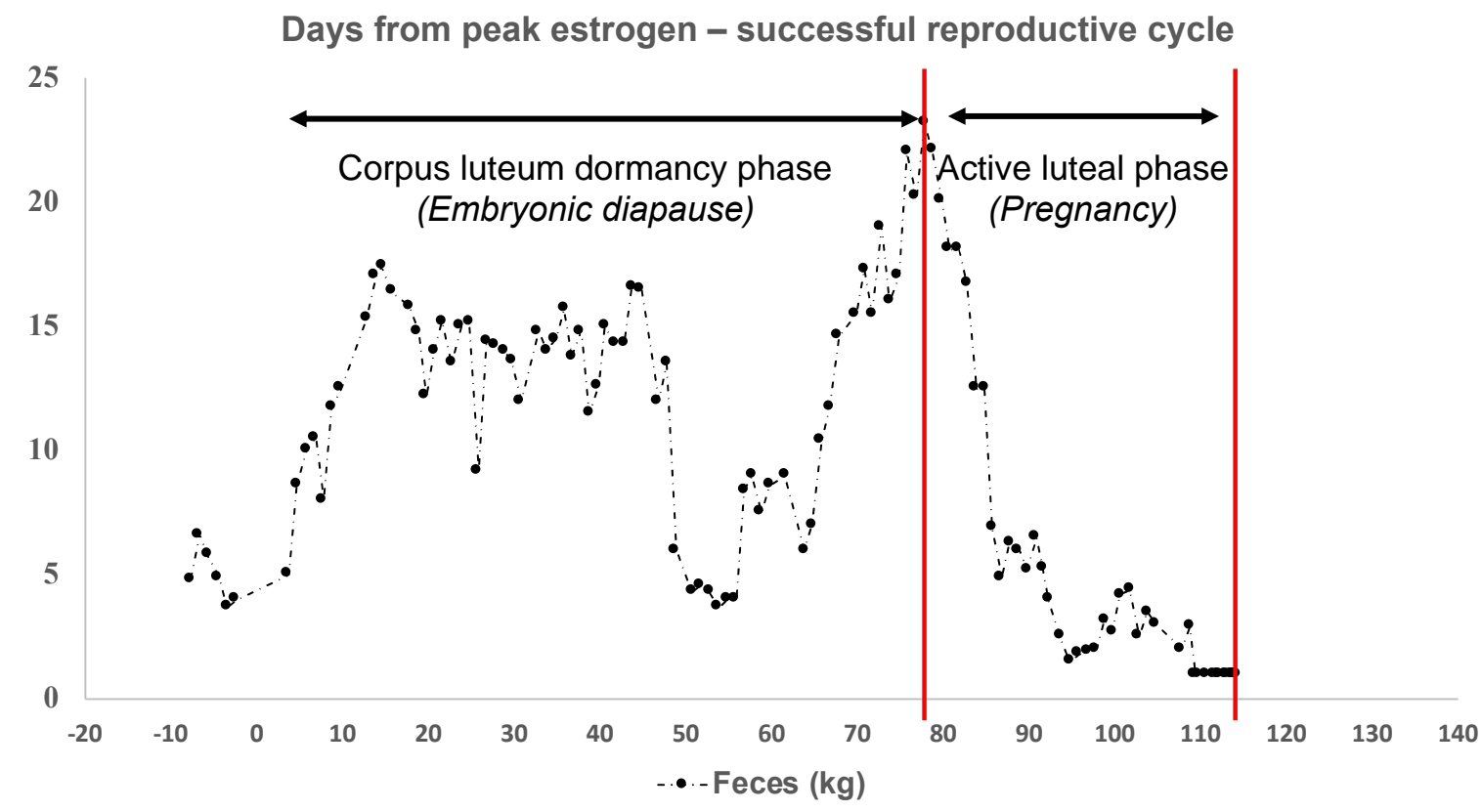

Figure 2 Overview of a successful cycle in a female giant panda. Fecal output was recorded as a measure of metabolism (expressed as daily fecal output in $\mathrm{kg}$ ). 
Secondly, to maintain a potential pregnancy, all females need the period of diapause to gain enough weight (energy resources) to survive the short pregnancy with the attendant exponential growth of the embryo [36]. Indeed, during this phase giant pandas sacrifice all energy to embryonic growth, and will mainly be inactive, instead of foraging for food.

For each giant panda, the metabolic turn-over point for corpus luteum reactivation will be individual and specific, depending on their condition and environmental factors before and after estrus, hence leading to different lengths in 'diapause' between and within individuals (see Figure 2).

In recent years, the giant panda population in captivity has been significantly growing. Nevertheless, not all pandas have been successful breeders, and genetically valuable animals may therefore be at risk of being under-represented. Additionally, despite the successful reproductive program in captive giant pandas during the past decades, surprisingly little is still known about the specific mechanisms regulating their reproductive systems.

In threatened species, the experimental access to matrices of interest (endometrium cells derived from different pregnancy stages plus the corresponding endometrium fluid and blastocysts) is very limited. However, with state-of-the-art assisted reproduction technologies (ART), such as ultrasound guided transcutaneous ovum pickup, being an option, the giant panda reproductive program could likely be lifted to the next level. As such, In vitro-fertilization of oocytes collected from genetically valuable females treated with follicle stimulating drugs and fertilized with sperm of (different) genetically underrepresented males could be pursued. In vitro early-stage blastocysts could then be transferred to any ovulated, but non-mated pseudopregnant foster female in the corpus luteum dormancy phase. Transferring more developed blastocysts would be most likely more challenging, as the development stage of the blastocyst needs to be synchronized with a correlating degree of reactivation in the female. As yet, the information on how a blastocyst is potentially evolving during late diapause/ early reactivation is lacking in giant panda, and optimal timing for potential embryo transfer should thus be defined by further research.

Inducing reactivation to synchronize embryo transfer is most likely not ideal in this species, as success of pregnancy will depend on the metabolic state of the female, with reactivation before gaining enough energetic resources potentially leading to failed attachment or impaired embryonic development.

The forthcoming applied research would obviously benefit from more basic research on reproductive biology in giant pandas or related carnivorous species. Nevertheless, previous reports describing in vitro oocyte maturation in giant pandas. as well as successful embryo recovery and transfer during delayed implantation in other bear species [37-39], allude to the validity of introducing the described ART concept to the giant panda captive breeding program.

\section{Conclusion}

ART is highly relevant to support breeding programs in wildlife species, particularly those at risk of extinction. Unraveling the pathways behind arrest of embryonic development and its maintenance may facilitate the establishment of new approaches and tools in ART, such as diapause-induced embryo preservation as an alternative for cryopreservation for long-term storage of embryos. Specifically, in the giant panda, with numerous females preparing every breeding season for pregnancy, even in absence of mating or artificial insemination, diapause-based ART may offer enhanced success in the breeding program, with the opportunity to manage the genetic quality of the captive population.

Ovum-pick up and embryo-transfer experiments in giant pandas are indeed invasive, but may additionally assist in the future unraveling of the mechanism behind diapause, without sacrificing 
any tissue or cells and without any deleterious effect on the giant panda population. The approach to elucidate and use diapause in the giant panda may serve as a blueprint for genetic biodiversity management in other endangered species with diapause.

\section{Declaration of interest}

The authors declare that there is no conflict of interest that could be perceived as prejudicing the impartiality of this article.

\section{Funding}

This work did not receive any specific grant from any funding agency in the public, commercial, or not-for-profit sector. Nevertheless, graphical data and background knowledge was applied from research funded by the Pairi Daiza Foundation, Beauval Nature and Ghent University.

\section{References}

[1] Renfree MB and Fenelon JC. 2017 The enigma of embryonic diapause. Development 144 3199-3210.

[2] Fenelon JC and Renfree MB. 2018 The history of the discovery of embryonic diapause in mammals. Biology of Reproduction 99 242-251.

[3] van der Weijden V. 2019 Dynamic transcriptome changes during embryonic diapause and reactivation in the embryo and endometrial epithelium of the European roe deer (Capreolus capreolus). $3^{\text {rd }}$ International Symposium on Embryonic Diapause, 2-6 June, Ascona, Switzerland.

[4] Lopes FL, Desmarais JA and Murphy BD. 2004 Embryonic diapause and its regulation. Reproduction 128 669-678.

[5] Kamemizu C and Fujimori T. 2019 Distinct dormancy progression depending on embryonic regions during mouse embryonic diapause. Biology of Reproduction 100 1204-1214.

[6] Spindler RE, Renfree MB and Gardner DK. 1996 Carbohydrate uptake by quiescent and reactivated mouse blastocysts. Journal of Experimental Zoology 276 132-137.

[7] Fenelon JC, Banerjee A and Murphy BD. 2014 Embryonic diapause: development on hold. International Journal of Developmental Biology 58 163-174.

[8] Menzies BR, Hildebrandt TB and Renfree MB. 2020 Unique reproductive strategy in the swamp wallaby. PNAS 117 5938-5942.

[9] Renfree MB. 1981 Embryonic diapause in marsupials. Journal of Reproduction and Fertility Supplement 29 67-78.

[10] Shaw G and Renfree MB. 1986 Uterine and embryonic metabolism after diapause in the tammar wallaby, Macropus eugenii. Journal of Reproduction and Fertility 76 339-347.

[11] Shaw G. 1996 The uterine environment in early pregnancy in the tammar wallaby. Reproduction. Fertility and Development 8 811-818.

[12] Periera RM and Marques CC. 2008 Animal oocyte and embryo cryopreservation. Cell Tissue Banking 9 267-277.

[13] Prieto MT, Sanchez-Calabuig MJ, Hildebrandt TB, Santiago-Moreno J and Saragusty J. 2014 Sperm cryopreservation in wild animals. European Journal of Wildlife Research 60 851-864.

[14] Hermes R, Hildebrandt TB and Göritz F. 2018 Cryopreservation in rhinoceros - Setting a new benchmark for sperm cryosurvival. PLOS ONE 13 https://doi.org/10.1371/journal.pone.0200154. 
[15] Hildebrandt TB, Hermes R, Colleoni S, Diecke S, Holtze S, Renfree MB, Stejskal J, Hayashi K, Drukker M, Loi P, Goritz F, Lazzari G and Galli C. 2018 Embryos and embryonic stem cells from the white rhinoceros. Nature Communications 92589.

[16] Saragusty J, Diecke S, Drukker M, Durrant B, Ben-Nun IF, Galli C, Goritz F, Hayashi K, Hermes R, Holtze S, Johnson S, Lazzari G, Loi P, Loring JF, Okita K, Renfree M B, Seet S, Voracek T, Stejskal J, Ryder OA and Hildebrandt TB. 2016 Rewinding the Process of Mammalian Extinction. Zoo Biology 35 280-292.

[17] Comizzoli P and Holt WV. 2014 Recent advances and prospects in germplasm preservation of rare and endangered species. Advances in Experimental Medicine and Biology 753 331-356.

[18] Jewgenow K and Paris MCJ. 2006 Preservation of female germ cells from ovaries of cat species. Theriogenology 66 93-100.

[19] Lermen D, Bloemeke B, Browne R, Clarke A, Dyce PW, Fixemer T, Fuhr GR, Holt WV, Jewgenow K, Lloyd RE, Loetters S, Paulus M, Reid GM, Rapoport DH, Rawson D, Ringleb J, Ryder OA, Spoerl G, Schmitt T, Veith M and Mueller P. 2009 Cryobanking of viable biomaterials: implementation of new strategies for conservation purposes. Molecular Ecology 18 1030-1033.

[20] Pablos MTP, Saragusty J, Santiago-Moreno J, Stagegaard J, Goritz F, Hildebrandt TB and Hermes R. 2015 Cryopreservation of Onager (Equus Hemionus Onager) Epididymal Spermatozoa. Journal of Zoo and Wildlife Medicine 46 517-525.

[21] Saragusty J, Prieto MT, Courtiol A, Potier R, Goritz F, Hildebrandt TB and Hermes R. 2016 Sperm rescue in wild African elephants. Reproduction Fertility and Development 28 1433-1442.

[22] Fernandez-Gonzalez L and Jewgenow K. 2017 Cryopreservation of feline oocytes by vitrification using commercial kits and slush nitrogen technique. Reproduction in domestic animals Zuchthygiene 52 Suppl 2 230-234.

[23] Mandawala AA, Harvey SC, Roy TK and Fowler KE. 2016 Cryopreservation of animal oocytes and embryos: current progress and future prospects. Theriogenology 86 1637-1644.

[24] Canesin HS, Brom-de-Luna JG, Choi YH, Pereira AM, Macedo GG and Hinrichs K. 2018 Vitrification of germinal-vesicle stage equine oocytes: Effect of cryoprotectant exposure time on in-vitro embryo production. Cryobiology 81 185-191.

[25] Moreau GM, Arslan A, Douglas DA, Song J, Smith LC and Murphy BD. 1995 Development of immortalized endometrial epithelial and stromal cell lines from the mink (Mustela vison) uterus and their effects on the survival in vitro of mink blastocysts in obligate diapause. Biology of reproduction 53 511-518.

[26] Ptak GE, Tacconi E, Czernik M, Toschi P, Modlinski JA and Loi P. 2012 Embryonic diapause in conserved across mammals. PLoS ONE 7 e33027. https://doi:10.1371/journal.pone.0033027.

[27] Fernandez-Gonzalez L, Hribal R, Stagegaard J, Zahmel J and Jewgenow K. 2015 Production of lion (Panthera leo) blastocysts after in vitro maturation of oocytes and intracytoplasmic sperm injection. Theriogenology 83 995-999.

[28] Spindler RE, Renfree MB and Gardner DK. 1999 Mouse embryos used as a bioassay to determine control of marsupial embryonic diapause. Journal of experimental zoology 283 590-599.

[29] Zhang H, Li D, Wang C and Hull V. 2009 Delayed implantation in giant pandas: the first comprehensive empirical evidence. Reproduction 138 979-986.

[30] Kersey DC, Wildt DE, Brown JL, Snyder RJ, Huang Y and Monfort SL. 2010 Endocrine milieu of perioestrus in the giant panda (Ailuropoda melanoleuca), as determined by non-invasive hormone measures. Reproduction Fertility and Development 22 901-912.

[31] Wei F, Hu Y, Yan L, Nie Y, Wu Q and Zhang Z. 2014 Giant Pandas are not an evolutionary cul-de-sac: Evidence from multidisciplinary research. Molecular Biology and Evolution 32 4-12.

[32] Kersey DC, Wildt DE, Brown JL, Snyder RJ, Huang Y and Monfort SL. 2010 Unique biphasic progestagen profile in parturient and non-parturient giant pandas (Ailuropoda melanoleuca) as determined by faecal hormone monitoring. Reproduction 140 183-193.

[33] Goritz F, Hildebrandt TB, Jewgenow K, Wagner N, Hermes R, Strauss G and Meyer HHD. 1997 Transrectal ultrasonographic examination of the female urogenital tract in nonpregnant and pregnant captive bears (Ursidae). Journal of Reproduction and Fertility 51 303-312. 
[34] Dehnhard M, Hildebrandt TB, Knauf T, Jewgenow K, Kolter L and Goritz F. 2006. Comparative endocrine investigations in three bear species based on urinary steroid metabolites and volatiles. Theriogenology 66 1755-1761.

[35] Wilson KS, Wauters J, Valentine I, McNeilly A, Girling S, Li R, Li D, Zhang H, Rae MT, Howie F, Andrew $\mathrm{R}$ and Duncan WC. 2019. Urinary estrogens as non-invasive biomarker of viable pregnancy in the giant panda (Ailuropoda melanoleuca). Scientific Reports https://doi.org/10.1038/s41598-019-49288-6.

[36] Wauters J, Wilson KS, Bouts T, Valentine I, Vanderschueren K, Ververs C, Howie A F, Rae MT, Van Soom A, Li R, Li D, Zhang H and Vanhaecke L. 2018 Urinary specific gravity as an alternative for the normalisation of endocrine metabolite concentrations in giant panda (Ailuropoda melanoleuca) reproductive monitoring. PLOS ONE 13 https://doi.org/10.1371/journal.pone.0201420.

[37] Boone WR, Catlin JC, Casey KJ, Dye PS, Boone ET and Schuett RJ. 1999 Live birth of a bear cub following nonsurgical embryo collection. Theriogenology 51 519-29.

[38] Tsubota T, Takahashi Y, Kanagawa H and Gohda K. 1991 Embryo Recovery during Delayed Implantation in the Captive Hokkaido Brown Bear, Ursus-Arctos-Yesoensis. Journal of Veterinary Medical Science 53 141-2.

[39] Zhang MJ, Hou R, Zhang AJ, Zhang ZH, He GX, Li GH, Wang JS, Li SC, Song YF, Fei LS and Chen HW. 1998 In vitro maturation of follicular oocytes of the Giant Panda (Ailuropoda melanoleuca): a case report. Theriogenology 49 1251-5.

\section{Audience Discussion}

Pierre Comizzoli: Have you ever suspected, that for such a long pregnancy length as in the rhino or the elephant, gestation length, you maybe have embryonic diapause at the beginning?

Thomas Hildebrandt: Maybe Barbara can answer that, because we found diapause in elephants, which is not well published. The cycle of the elephant is 4 months and there is a huge morphologic difference between the male and the female. If there's a male around and the females comes into estrus it will be raped. So no decision on mating from the female side. But there's this cryptic female choice, because investment into the offspring in the elephant is a lifetime decision for the female. We saw by ultrasound that the first sign of implantation took place 55 days after artificial insemination, the advantage of using artificial insemination is that we know exactly the time of ovulation. So, the female elephant is using this prolonged period deciding to implant the embryo or not. Interestingly, the embryo has to survive a phase when the CL-size is declining, even if the female is pregnant, it looks like she's not pregnant and normally causes a lot of nervousness from the curators, and then suddenly grows and increases four times, we think that's induced by the crosstalk between the embryo and the maternal environment. So, elephants are most likely have a diapause. Rhinoceros we would say no, because we checked them after artificial insemination and we see an early embryonic vesicle.

Colin Stewart: So, from what I understand that there's a limitation on the availability of gametes ensuring the survival of those different species. However, there has been some success in producing gametes from cultured cell lines, particularly from mice and even humans. I was wondering isn't it much easier to preserve rhino fibroblasts and turn them into stem cells and from that produce gametes. How much work has been done to see if that's an alternative?

Thomas Hildebrandt: I had that in my presentation, but due to time constraint I took it out. We have a very close collaboration with a group in Japan, Prof Katsuhiko Hayashi. We formed a consortium where we produced ES-cells from rhinos and we also have iPS-cells from northern white rhinos. We think that's the only way to maintain genetic diversity. These two females will produce offspring, but that the offspring will never restore an entire population. So, we need to work with fibroblast cell frozen already from other individuals to produce the artificial gametes as well as the natural gametes. However, this will take time and we have to overcome a lot of obstacles. Thus, we want to have the in vitro system with natural gametes in place. This is our 
learning platform to perform IVF and produce embryos, before we are able use the artificial gametes. We bank fibroblast cells from every single rhino we get our hands on, because we know now, that you can transform any cell into an embryonic cell. So that's revolutionizing the entire conservation approach.

Katarina Jewgenow: Are there any attempts to flush embryos out of pandas? It might be easier to start the experiments with flushed embryos. I just thought because somebody said that there are so many pandas around.

Thomas Hildebrandt: I would think it's a no-go. You can't do such invasive techniques, because the reproductive part of the female giant panda would most likely require a surgical intervention to do flushing in an optimal way and therefore it is not on the list. However, to harvest oocytes on infertile females, that is much more likely.

Susanne Ulbrich: I found it interesting how you displayed the breeding efficiency or offspring number in the wild related to livestock and related to endangered species. Regarding the Gaussian curve you drew in the middle, do you think it is the same for the different species regarding female and male around, because from the female point of view, it might be very inefficient to be a bad breeder, so probably these species have never ever survived evolution. So, I wonder whether the drawing you made was hypothetically correct specifically for the male, but rather not for the female. Would you agree on the mammal side?

Thomas Hildebrandt: No, I would agree on the opposite. Females are more sensitive for having no reproductive success, because of asymmetric reproductive aging. So, if a female accumulates too many cycles without getting pregnant, we see dramatic pathology on the uterus and on the ovaries. However, some of the candidates we had in our hands, the Sumatran rhino for example, were caught in palm tree plantations and they had most likely a lot of intake of pesticides and their sperm count is very bad. So, there are quite a lot of complex infertility parameters, some natural, some artificial. If you work with a critically endangered species it is critically endangered because it has problems with reproduction, it's not an ideal breeder.

Grazyna Ptak: I have a question related to the blastocysts of roe deer. Did you try to use different laboratories with the expertise in embryo freezing.

Katarina Jewgenow: The problem is we don't get access to roe deer embryos quite often.

Grazyna Ptak: So, nobody here present is able to answer how these embryos are surviving?

Katarina Jewgenow: Actually, the embryos we froze for Susanne, we just left them in culture medium for one hour after freezing to check the expansion, because Susanne wanted to be sure that the embryos were alive. So, I am convinced that they survived the freezing procedure. So, I guess that there were some little differences between the laboratories, or even during the transportation to Italy something went wrong. whatever...

Thomas Hildebrandt: The technique was done in Germany by Cesare Galli, the world expert on embryo freezing. 